\title{
High Efficiency with Small Coils Area Ratio of Magnetic Resonant Wireless Power Transfer System
}

\author{
Thabat Thabet, and John Woods
}

\begin{abstract}
Wireless power transfer using magnetic resonance requires cutting flux lines generated from the transmitter coil by the receiver coil. This letter shows that an exact one to one coil area ratio or CAR (i.e. primary relative to secondary) is not a pre-condition to obtain high efficiency. It is also shown that high efficiency can be achieved for relatively small CARs by adjustment of the turns ratio. We go on to show that it is possible to achieve a higher energy efficiency than the coil area ratio and the associated flux cut would dictate.
\end{abstract}

Index Terms-Coil Area Ratio; High Efficiency; Magnetic Resonant; Mutual Inductance; Wireless Power Transfer

\section{INTRODUCTION}

The basis of near field wireless power transfer between any two coils is the process of magnetic induction. Passing an alternating current through the first coil (transmitter), produces a magnetic field around it, and cutting the produced magnetic flux lines by the second coil (receiver) creates an electromotive force across it [1]. The ratio between the cross sectional areas of the receiver coil relative to the transmitter coil (Coil Area Ratio CAR) indicates the percentage of flux lines that are cut by the receiver.

In a normal transformer, the primary coil produces the magnetic field and the secondary coil cuts all the flux line by virtue of an iron core. Therefore, transformers have nearly one hundred percent efficiency because of the high coupling. However, without the iron core, the coupling is quite small and it is difficult to achieve high efficiency.

A promising technology to achieve wireless energy transfer at high efficiency through large distance is magnetic resonance [2], [3]. That is carefully choosing a capacitor for each coil/inductor (transmitter and receiver) so they resonate at the same frequency $f_{r}$ as [4]:

$f_{r}=\frac{1}{2 \pi \sqrt{L_{1} C_{1}}}=\frac{1}{2 \pi \sqrt{L_{2} C_{2}}}$

where $L_{1}, C_{1}, L_{2}$ and $C_{2}$ are the inductance and the capacitor of the transmitter and the receiver, respectively as shown in Fig. 1. The figure also shows equivalent circuit of a magnetic resonant wireless power transfer system where $V_{S}$ and $R_{S}$ are the voltage sources and the internal resistor, and $R_{2}$ is the load resistor.

Published on October 15, 2018.

T. Thabet and J. Woods are with School of Computer Science and Electronic Engineering, Essex University, Wivenhoe Park, Colchester CO4 3SQ, UK (tfytha@essex.ac.uk and woodjt@essex.ac.uk)

T. Thabet is also with Engineering Technical College of Mosul/ Iraq.

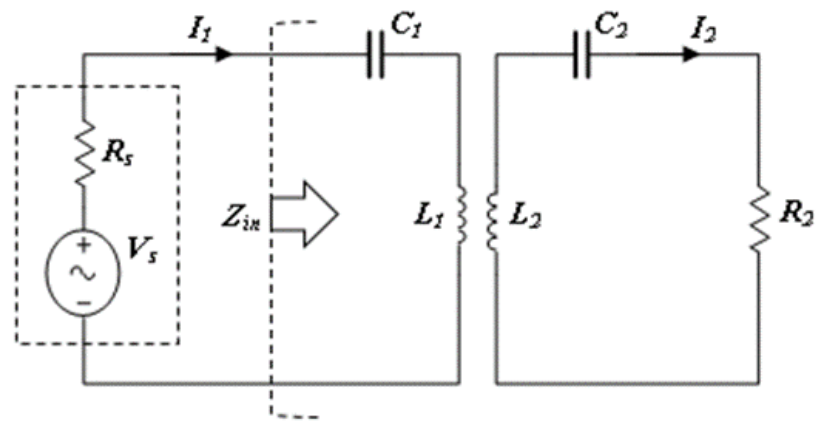

Fig. 1. Equivalent circuit of a magnetic resonant wireless power transfer system

Transfer of power between two coils with a large cross sectional area can achieve higher efficiency than the same transmitter and a smaller receiver. This is obvious because of the coils area ratio and the percentage of flux lines being cut by the receiver. However, it is possible to obtain high efficiency even with small CARs. This letter focuses on how to increase the efficiency of the resonant power transfer with a relatively small CAR.

\section{ANALYSIS OF THE SYSTEM}

This can be achieved by applying Kirchhoff's law of voltage on each loop in Fig. 1 in order to express the circuit as following [5]:

$\left[\begin{array}{c}V_{S} \\ 0\end{array}\right]=\left[\begin{array}{cc}R_{S}+j X_{L 1}-j X_{C 1} & -j X_{M} \\ -j X_{M} & R_{2}+j X_{L 2}-j X_{C 2}\end{array}\right]\left[\begin{array}{l}I_{1} \\ I_{2}\end{array}\right]$

where $M$ is the mutual inductance between the two coils, $X_{M}$ $=2 \pi f M, X_{L}=2 \pi f L$ and $X_{C}=1 / 2 \pi f C, I_{1}$ and $I_{2}$ are the currents in the first and second loops respectively.

Under the concept of maximum transfer of power, which requires $Z_{\text {in }}=R_{S}$, the efficiency eff can be calculated as:

eff $=\frac{P_{\text {out }}}{P_{\text {in, } \max }}$

where $P_{\text {out }}$ and $P_{i n, \max }$ are the output and maximum input power; and calculated as:

$P_{\text {out }}=\frac{1}{2}\left|I_{2}\right|^{2} R_{2}$

$P_{i n, \max }=\frac{V_{S}^{2}}{8 R_{S}}$ 


\section{IDENTICAL COILS}

That is a one to one coil area ratio.

1) Starting with a set of two identical resonators as transmitter and receiver (parameters shown in Table I Combination1), gives the maximum efficiency at a $10 \mathrm{~cm}$ gap between the two coils as shown in Fig. 2 (Comb1). The figure presents the efficiency versus distance. The axial movement of the coupled coils degrades the power transfer efficiency [6]. Fig. 3 shows through the efficiency versus frequency that the maximum transfer of power is at the resonant frequency. Note that Th refers to the theoretical results, Prac refers to the experimental results and Comb refers to the Combination. The mutual inductance between them is calculated as [7] and it depends on the size of the coils (including the cross sectional area and the number of turns).

TABLE I: PARAMETERS OF COMBINATIONS

\begin{tabular}{|c|c|c|c|c|}
\hline Parameters & 1 & 2 & 3 & 4 \\
\hline$C(\mathrm{pF})$ & 188 & 550 & 266 & 174 \\
\hline$L(\mu \mathrm{H})$ & 31.3 & 10.8 & 21.6 & 33.2 \\
\hline No. of turns & 8 & 16 & 16 & 32 \\
\hline Diameter of the coil $(\mathrm{cm})$ & 24.5 & 6 & 8.4 & 6 \\
\hline
\end{tabular}

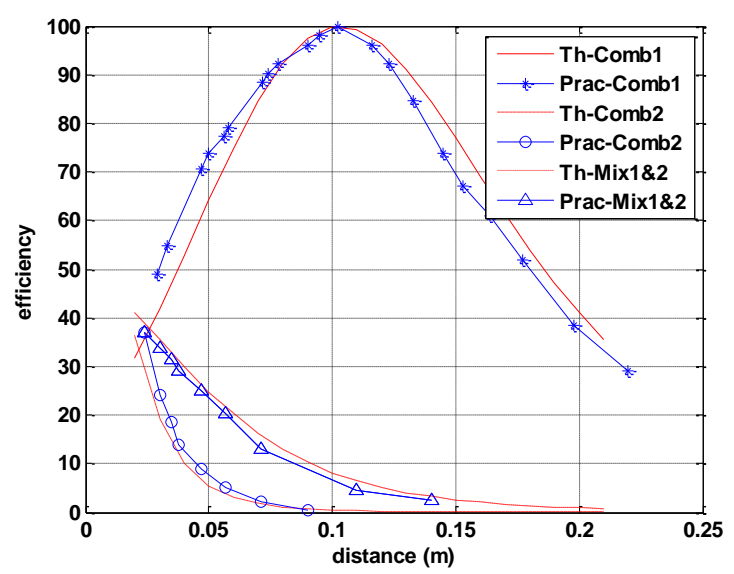

Fig. 2. Efficiency versus distance at resonant frequency

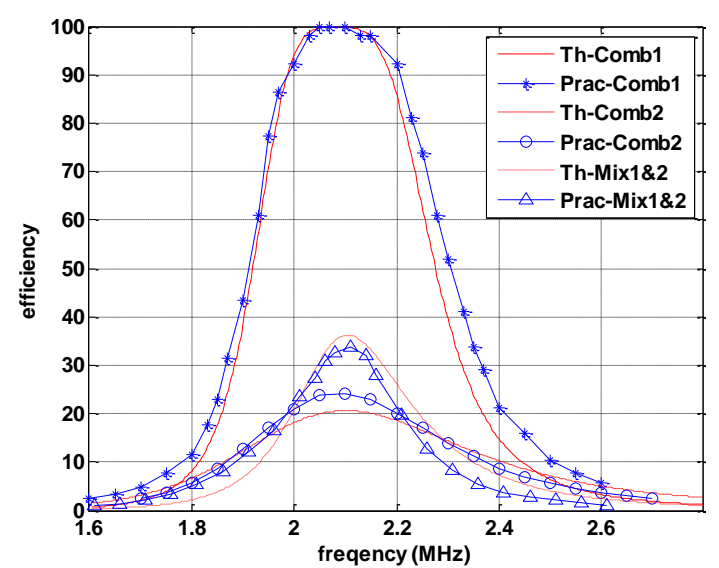

Fig. 3. Efficiency versus frequency at gap $=10 \mathrm{~cm}$ for $\mathrm{comb} 1$ and $3 \mathrm{~cm}$ for comb2 and mix $1 \& 2$

2) Replacing the two resonators with another set (the characteristics of them are shown in Table I Combination 2) to work at the same frequency, provides different curves of efficiency versus distance and frequency as shown in Fig. 1 and 2 (Comb2).

The results are initially surprising, lower efficiency occurs for a $3 \mathrm{~cm}$ gap, in spite of near full flux being intersected for both cases. The reason is the difference in the mutual inductance which depends on the size of the two coils.

\section{DIFFERENT SIZE COILS}

In this part, a small CAR is studied by using one resonator from the first set as a transmitter and one from the second set as a receiver with a $3 \mathrm{~cm}$ gap. As expected the system delivers less power than the first case of identical resonators because of the small CAR $(1: 17)$. Yet the power transferred is greater than the second case even though there is a small CAR; this is because of the higher mutual inductance between the coils. The two curves, efficiency versus distance and frequency, are shown in Fig. 2 and 3 (mix 1\&2). Note that mix refers to the mixed size coils.

\section{INCREASE THE EFFICIENCY}

Changing the receiver resonator of the last section with:

1) A coil which has double the cross section area and a suitable capacitor to resonate at the same frequency (Table I Combination3), leads to higher efficiency than Fig. 2 and 3 (mix $1 \& 2)$. Fig. 4 and 5 (mix1\&3) shows efficiency versus distance and frequency. CAR is (2:17).

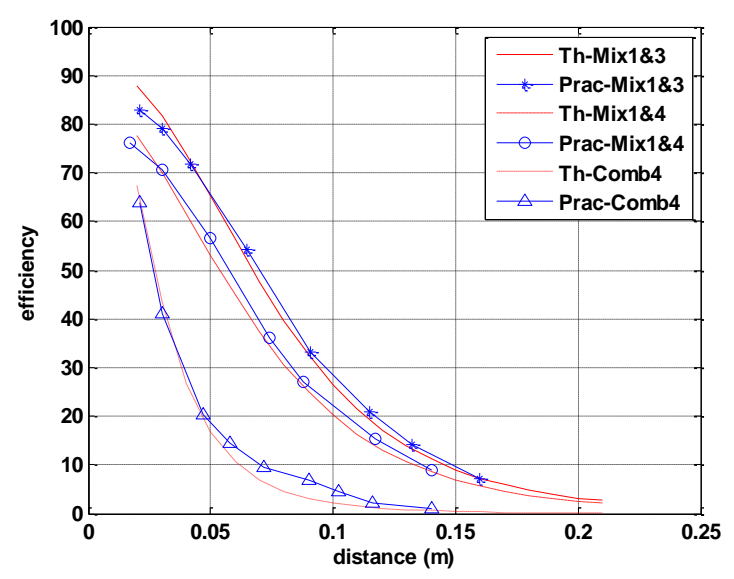

Fig. 4. Efficiency versus distance at resonant frequency

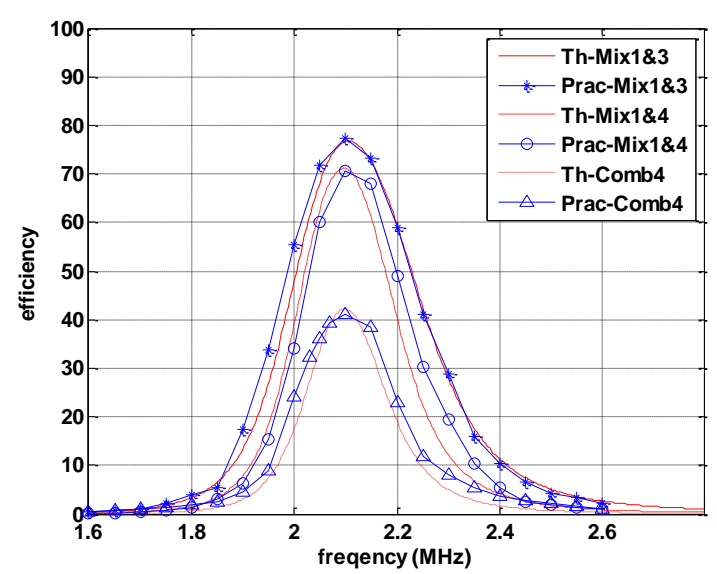

Fig. 5. Efficiency versus frequency at gap $=3 \mathrm{~cm}$ 
2) A coil which has twice the number of turns and a suitable capacitor to resonate at the same frequency, leads to higher efficiency than Fig. 2 and 3 $(\operatorname{mix} 1 \& 2)$. The parameters of the new resonator are shown in (Table I Combination4) and the related results are shown in Fig. 4 and 5 (mix $1 \& 4)$.

3) Using the resonators shown in Table I Combination 4 as transmitter and receiver, increases the efficiency, as shown in Fig. 4 and 5 (Comb4) compared with the second step of identical resonators section Fig. 2 and 3 (Comb2).

As shown in Fig. 4 and 5, higher efficiency can be achieved by increasing the number of turns in the receiver as well as increasing the cross section area (doubling in this experiment). Therefore, we have shown that it is possible to achieve a higher energy transfer than the CAR and the associated flux cut would dictate.

\section{CONCLUSION}

The efficiency of magnetic resonant wireless power transfer is controlled by the mutual inductance between the coils. Several factors affect the mutual inductance, such as the size of the coils including the cross section area and the number of turns. It is possible to increase the efficiency by increasing one or both of them.

Higher efficiency can be achieved with small section coils or with small CARs by increasing the number of turns in order to increase the mutual induction between them. The limitation is the diminishing size of the capacitor to resonate at the desired frequency. We have shown that it is possible to achieve a higher energy transfer than the CAR and the associated flux cut would dictate.

\section{REFERENCES}

[1] A. Mahmood, A. Ismail, Z. Zaman, H. Fakhar, Z. Najam, M. Hasan, and S. Ahmed, "A Comparative Study of Wireless Power Transmission Techniques," Journal of Basic and Applied Scientific Research, vol. 4, no. 1, pp. 321-326, 2014.

[2] H.-G. Park, J.-H. Jang, J.-H. Kang, H. D. Tiwari, Y.-K. Moon, and K.-Y. Lee, "Automatic power controlled, load compensated magnetic resonant wireless power transmitter," International Journal of Electronics Letters, vol. 2, no. 2, pp. 121-133, 2014.

[3] P. Vuori, J. Kari, and J. Muurinen, "Method and apparatus for wireless power transfer," Google Patents, US9859719B2, 2018.

[4] C. Balanis, ANTENNA THEORY Analysis and Design, 2nd ed., Canada: Wiley, 1997.

[5] T. Thabet, and J. Woods, "An Approach to Calculate the Efficiency for an N-Receiver Wireless Power Transfer System," IJACSA, vol. 6, no. 9, pp. 91-98, 2015.

[6] T. I. Anowar, S. D. Barman, A. Wasif Reza, and N. Kumar, "Highefficiency resonant coupled wireless power transfer via tunable impedance matching," International Journal of Electronics, pp. 1-19, 2017.

[7] A. C. M. de Queiroz, "Mutual inductance and inductance calculations by Maxwell's Method," Home page of Dr. Antonio Carlos M. de Queiroz, 2005.

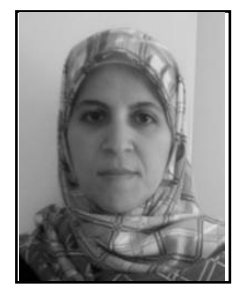

Thabat Thabet (S'16) received the B.S. and M.S degrees in electrical engineering/ electronics and communications from the University of Mosul, Iraq, in 1991 and 1997, respectively.

She is working as a lecturer assistant in the Engineering Technical College of Mosul/ Iraq. She is currently a Ph.D. student in the School of Computer Science and Electronic Engineering (CSEE), University of Essex, Colchester.

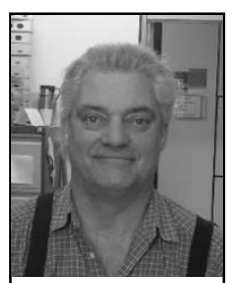

John Woods received the B. Eng. and Ph.D. degrees in the School of Computer Science and Electronic Engineering (CSEE), University of Essex, Colchester, UK, in 1996 and 1999 respectively.

$\mathrm{He}$ is a senior lecturer within the same department where he has worked for the last 20 years. His interests include image processing: segmentation, tracking, traffic modelling and image coding. He also has interests in robotics, autonomous flying vehicles and sensor networks. It is also a particular interest in wireless power transfer for sensors that has spawned this contribution.

Dr. Woods is a member of the world renowned Essex Robotic group. He has been involved in a number of EPSRC, TSB and EU funded research projects relating to image processing, control systems networking and remote control and configuration. 